\title{
LES CRISTAUX LIQUIDES NÉMATIQUES
}

\author{
P.-G. de GENNES \\ Laboratoire de Physique des Solides \\ Bâtiment 510, Université Paris 11, 91, Orsay \\ (Texte transcrit à partir de l'enregistrement en séance)
}

\begin{abstract}
Résumé. - On discute la rupture de symétrie et la nature du paramètre d'ordre dans un fluide nématique ainsi que leurs conséquences sur les modes collectifs de grande longueur d'onde, les singularités de l'alignement et les effets de champs extérieurs.
\end{abstract}

Abstract. - The broken symmetry and the nature of the order parameter in a nematic fiuid are discussed. The collective modes, the singularities of the alignment and the effects of external fields are presented from this view point.

Je suis embarrassé de vous parler de ce sujet d'une part parce que c'est un sujet vaste, que je ne domine pas du tout, d'autre part parce que j'ai une bonne grippe et mon élocution va s'en ressentir. Tout de même c'est un sujet suffisamment interdisciplinaire pour être abordé dans une réunion comme celle-ci. Je vais choisir parmi les cristaux liquides le groupe le plus courant, qui est ce qu'on appelle les nématiques.

La figure 1 représente le type d'arrangement des molécules dans un fluide nématique. Les molécules sont plus ou moins parallèles les unes aux autres et elles sont allongées en forme de bâtons. La figure 2 donne des exemples chimiques de tels petits bâtons. Ce sont des molécules d'environ $25 \AA$ de long, d'environ $5 \AA$ de large et assez rigides, avec aux bouts de courtes chaînes un peu plus flexibles qui l'aident à rester en phase liquide.

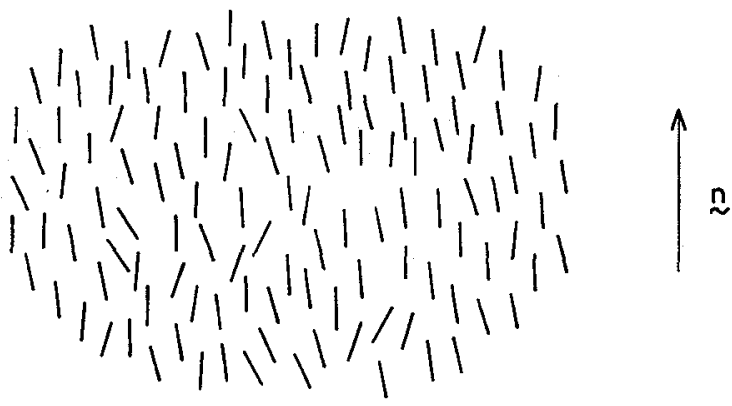

Fig. 1. - Disposition des molécules dans un fluide nématique.

Si je prends des molécules de ce genre, si je les chauffe, au lieu de passer directement du solide à un liquide usuel isotrope, entre les deux je trouve la phase intermédiaire de la figure 1. Cette phase est liquide, elle coule admirablement, elle a une viscosité comparable à celle de l'eau mais, quoique liquide, elle a des propriétés, optiques étonnantes, du fait que les axes moléculaires sont plus ou moins parallèles à une même direction; le matériau est optiquement uniaxe.<smiles>COC1CCC(N=N[N+]([O-])C2CCC(OC)CC2)CC1</smiles>

Paraazoxyanisole<smiles>CCCCC1CCC(N=CC2CCC(OC)CC2)CC1</smiles>

MBBA

FIG. 2. - Exemples de matériaux donnant une phase nématique.

Historiquement, ces phases nouvelles ont été découvertes il y a plus de 70 ans, mais on a mis très longtemps à se convaincre qu'il s'agissait d'un phénomène fondamental. Au début, beaucoup de gens pensaient qu'il s'agissait de petits cristallites mal dissous ou plus généralement d'un système hétérogène. Progressivement, on a réalisé qu'il s'agissait au contraire d'une phase fondamentale douée d'une symétrie très rigide ; cette contribution est d'ailleurs due au grand-père de notre président; c'est Georges Friedel qui, dans les années 1910 à 1925, par des études essentiellement faites au microscope avec des moyens matériels extrêmement légers et une très bonne intelligence géométrique du problème, a réussi à classer ces types de matériaux, à définir leur symétrie de façon rigoureuse. Ceci m'amène à discuter la symétrie de cette phase qui est à la fois liquide et anisotrope. 
Nature du paramètre d'ordre. - On est tenté de caractériser l'alignement par un vecteur. Sur la figure 1, on a fait apparaître un vecteur $\mathbf{n}$ unitaire qui définit l'axe optique et qui est couramment appelé le directeur du nématique. Toutefois, cette notation est un peu dangereuse parce qu'elle suggère que la direction vers le haut et la direction vers le bas ne jouent pas le même rôle. En fait, l'expérimentation nous montre que ces deux directions sont équivalentes. Si chaque molécule porte un dipôle électrique permanent, dans l'état nématique, en l'absence de contraintes extérieures, il y a autant de dipôles vers le bas que de dipôles vers le haut; le matériau n'est pas ferroélectrique. Donc le choix d'un vecteur pour caractériser l'état du système n'est pas très bon. Ce qui définit vraiment l'ordre nématique, c'est le fait que par exemple la constante diélectrique est un tenseur anisotrope. Par conséquent, ce qu'on peut utiliser comme paramètre d'ordre pour caractériser ce système, c'est un tenseur symétrique de rang 2, $Q_{\alpha \beta}$, qui est la partie anisotrope de la constante diélectrique à une fréquence de référence. $Q_{\alpha \beta}$ est un tenseur symétrique, de trace nulle; si on se place dans les axes propres de la matrice $Q_{\alpha \beta}$, on imagine tout de suite deux types de phases possibles :

a) La phase la plus générale correspond à une situation où le tenseur a trois valeurs propres distinctes, correspondant à un nématique biaxe.

b) Une phase plus particulière, où on a deux valeurs propres identiques : un nématique uniaxe. La nature ne nous fournit que des uniaxes. Il est possible qu'un jour elle nous fournisse des biaxes, mais ce n'est pas sûr.

Ce paramètre d'ordre mérite qu'on y réfléchisse un peu, parce qu'il est assez différent de ceux que nous sommes habitués à rencontrer. Discutons donc en parallèle quelques exemples où l'on a une transition ordre-désordre qui correspond à la rupture d'un groupe continu de symétrie. C'est un mot un peu savant mais essayons de le rendre concret.

Le premier exemple est un ferro magnétique de Heisenberg; le paramètre d'ordre est l'aimantation M. C'est un vecteur qui peut pointer dans n'importe quelle direction (paramètre d'ordre: vecteur à trois composantes). Le deuxième exemple est celui des supraconducteurs, dans lequel le paramètre d'ordre est la fonction d'onde des paires de Cooper : c'est un scalaire complexe $\psi$. Le troisième exemple est celui des nématiques dans lesquels le paramètre d'ordre est un tenseur symétrique de rang 2, réel. Les propriétés de symétrie sont très différentes pour les trois cas.

a) Dans le cas du ferromagnétique, si je construis l'énergie libre, en fonction de ce vecteur $\mathbf{M}$, elle est inchangée si je renverse les aimantations. Ceci, fondamentalement, c'est une propriété liée au renversement du sens du temps. L'hamiltonien des spins en interaction est invariant par le renversement de $t$ et le renversement de $t$ change $\mathbf{M}$ en $-\mathbf{M}$. A partir de cette propriété de symétrie, un raisonnement dû à Landau montre qu'il est possible d'avoir une transition du second ordre, depuis une phase ordonnée ferro magnétique jusqu'à une phase désordonnée, paramagnétique.

b) Pour les supraconducteurs, on a $F(-\psi)=F(\psi)$. La motivation est assez différente : ici, il s'agit d'une transformation de jauge ; on peut toujours multiplier $\psi$ par un facteur de phase sans changer les propriétés observables du système. Ce facteur de phase peut être pris égal à $\pi$, d'où la propriété ci-dessus. De la même façon, on conclut que la transition d'un état ordonné, où il $\mathrm{y}$ a un $\psi$ macroscopique (donc superfluidité) jusqu'à un état désordonné $(\psi=0)$ peut se permettre d'être du deuxième ordre.

c) Passons maintenant au cas nématique, et regardons la dépendance de l'énergie libre, pour un uniaxe, par rapport à la valeur propre principale $\mathrm{du}$ tenseur $Q_{\alpha \beta}$. Cette énergie libre n'a aucune raison d'être la même si on change cette valeur propre en son opposée. Physiquement, changer cette valeur propre en son opposée voudrait dire que l'on passe d'un ellipsoïde des indices allongé à un ellipsoïde aplati. Il n'y a aucune correspondance simple entre ces deux états. Donc, ici, pas de symétrie dans l'énergie libre en fonction du paramètre d'ordre. Ceci permet de démontrer immédiatement que la transition, depuis la phase ordonnée (nématique) jusqu'à la phase désordonnée (liquide isotrope), doit être du premier ordre. Expérimentalement, c'est bien ce qu'on observe ; toutes les transitions nématiques isotropes sont du premier ordre. Elles sont d'ailleurs faiblement du premier ordre. Les chaleurs latentes sont, disons, dix fois plus faibles qu'à la fusion. La discontinuité de volume à la transition est de l'ordre de $1 \%$; ceci a été établi en premier par $M$. Bauer avec Bernamont vers 1935.

Les « variables de phase ». -- Maintenant, regardons ce qui se passe dans la phase ordonnée, en dessous de la transition ordre-désordre. Ici, il y a une notion que je suis loin de comprendre entièrement du point de vue de la théorie des groupes, mais qui est très importante : c'est ce que j'appellerai la notion de description réduite. Commençons par un ferromagnétique d'Heisenberg. Quand nous sommes en dessous du point d'ordre, ce ferro magnétique a une grande aimantation. L'aimantation a une longueur $M$ qui est assez bien fixée (à une température donnée). Il y a de petites fluctuations de la longueur, mais elles ne sont pas très anormales. Par contre, ce qui n'est pas du tout fixé, c'est la direction de l'aimantation. En l'absence de force extérieure, elle peut tourner dans tous les sens. Ceci suggère une description réduite dans laquelle on néglige les fiuctuations de la longueur et on décrit l'état du système, par exemple par 
deux angles $\theta$ et $\varphi$ qui donnent l'orientation de l'aimantation. Ces deux angles $\theta$ et $\varphi$, c'est ce qu'on pourrait appeler les variables de phase du système et eux, peuvent avoir des fluctuations absolument géantes. Même chose pour un supraconducteur. Dans l'état superfluide, le module du paramètre d'ordre est pratiquement fixé. Il a des fluctuations, mais peu pathologiques. Au contraire, la phase de $\psi$ fluctue énormément. Dans les nématiques, nous avons la même chose et nous pourrions repérer la situation par deux angles $\theta$ et $\varphi$ pour un nématique uniaxe, ou par trois angles d'Euler pour un nématique. Limitons-nous aux uniaxes, puisque ce sont les seuls qu'on connaisse. La description réduite implique donc deux angles, ou bien un vecteur unitaire, le directeur $\mathbf{n}$.

Comme je l'ai dit, les variables de phase ont des fluctuations géantes; ceci se traduit par des propriétés remarquables pour la plupart de ces systèmes.

a) Pour le cas magnétique, les fluctuations de la direction de l'aimantation dans la phase ordonnée s'observent de façon optimale par diffusion magnétique des neutrons. Leur dynamique qui est régie par la loi de dispersion des ondes de spin. Pour un vecteur d'onde $q$ donné, la fréquence d'oscillation est $\omega_{q} \sim q^{2}$. En particulier, pour $q \rightarrow 0, \omega_{q} \rightarrow 0$.

b) Pour un superfluide comme l'hélium, les fluctuations de la phase de $\psi$ sont associées à des fluctuations de courant longitudinal, donc à des ondes sonores $\left(\omega_{q} \sim q\right)$. Dans ce cas aussi, pour $q \rightarrow 0, \omega_{q} \rightarrow 0$. D'une façon plus générale, dans un système où les forces sont à courte portée, il suffit de très peu d'énergie pour créer une modulation spatiale lente des variables de phase $=$ pour $q \rightarrow 0$ le spectre des excitations se concentre dans la région de fréquence nulle.

c) Cette remarque s'applique aux nématiques, mais ici le contexte est assez différent: nous avons un liquide, dans lequel les effets d'amortissement visqueux sont prédominants. Au lieu d'avoir des fréquences réelles d'excitation $\omega_{q}$, nous avons des fréquences purement imaginaires $i / \tau_{q}$. La vitesse de relaxation $1 / \tau_{q}$ est en gros de la forme

$$
\frac{1}{\tau_{q}}=\text { Cte. } q^{2} \text {. }
$$

Comment peut-on observer les fluctuations d'orientation et leur relaxation?

Une modulation du vecteur $\mathbf{n}$ est une modulation spatiale de la direction de l'axe optique. Elle cause donc une diffusion de la lumière qui est très facilement observable. L'effet est très intense puisqu'une fluctuation de grande longueur d'onde demande une très faible énergie : son amplitude est très grande à l'équilibre thermique. Nous voyons apparaître là une propriété frappante des cristaux liquides; ils se prêtent souvent beaucoup mieux à l'expérimentation (et à l'étude des symétries brisées) que les matériaux magnétiques ou superfluides.
La conclusion pratique, c'est que les nématiques sont turbides si on les regarde sous des épaisseurs macroscopiques (à l'échelle de quelques millimètres d'épaisseur). C'est une des raisons pour lesquelles on a longtemps cru que c'étaient des matériaux hétérogènes. En fait, il s'agit d'une propriété intrinsèque. Les intensités de diffusion de la lumière ont été étudiées assez tôt par de belles expériences de P. Chatelain à Montpellier (vers 1948).

$\mathrm{Du}$ point de vue répartition en fréquences, il faut voir que les $1 / \tau_{q}$ que je cite ici, pour des $q$ (vecteurs d'onde) dans le domaine optique, sont typiquement dans le domaine du kilocycle : c'est-à-dire que les expériences n'ont été réalisables que tout récemment par des méthodes de battement de photons.

Ces expériences ont été faites en particulier par le groupe d'Orsay. On a pu en tirer des informations assez remarquables sur les énergies de distorsion et surtout sur les mécanismes de friction. L'analyse théorique de la friction est relativement subtile. Il s'agit de déterminer la dynamique de systèmes où il y a, d'une part, des écoulements possibles puisque c'est un liquide et, d'autre part, un degré de liberté interne, la direction d'orientation. Cette hydrodynamique a été écrite en premier par un groupe américain (Ericsen, et son élève Leslie). Le système est décrit non pas par une viscosité mais par 5 coefficients de friction. (En fait, il y en avait 6 au départ, puis Parodi a établi qu'il y avait entre eux une certaine relation d'Onsager qui les réduit à 5.) On a pu déterminer au stade actuel de l'expérimentation 4 de ces 5 coefficients de friction par les études de diffusion inélastique. Vous voyez que les fluctuations sont donc un moyen d'investigation assez utile dans ces systèmes.

Ces fluctuations seraient bien intéressantes aussi à étudier dans des systèmes à deux dimensions. On sait, par exemple, que dans un ferro magnétique de Heisenberg à deux dimensions, les fluctuations sont divergentes, et il n'y a pas d'ordre à longue distance. Il y a localement des régions ordonnées mais, si on change de point d'observation, on trouve que la direction d'alignement n'est plus la même. Expérimentalement, on a essayé d'observer ces propriétés sur des cristaux où les ions magnétiques sont disposés en couches assez espacées. Mais les interactions entre couches, et aussi les énergies d'anisotropie à l'intérieur d'une couche, compliquent la situation : le problème sera discuté ici en détail par B. Jancovici. Avec les nématiques, nous avons peut-être le moyen de faire mieux. Ceci est une idée qui nous est venue à la suite de discussions avec un expert des films superficiels $\left(\mathrm{M}^{\mathrm{me}} \mathrm{L}\right.$. Terminassian-Saraga).

A un interface eau-air, je mets des molécules qui contiennent une chaîne carbonée rigide (peut-être avec quelques doubles liaisons pour la rendre bien rigide). Aux deux bouts de la molécule, nous avons des groupes hydrophiles (par exemple des fonctions alcool) pour assurer que la molécule se dispose hori- 
zontalement à l'interface eau-air. Si on parvenait à faire un film monomoléculaire, liquide, de ce type, on devrait dans certains cas réaliser à la surface de l'eau un nématique bidimensionnel. Ce système devrait nous montrer de façon tout à fait rigoureuse l'ordre anormal ou «quasi-ordre» obtenu à deux dimensions. Il semble, par des calculs théoriques candides, qu'on devrait pouvoir étudier la diffusion de la lumière par un tel film, et observer des lois tout à fait anormales. Il faut mentionner une difficulté : il $\mathrm{y}$ a une autre source de diffusion à un interface qui a été largement étudiée par $\mathrm{M}$. A. Bouchiat et ses collaborateurs; c'est le fait que la surface ondule; ceci donne une diffusion un peu plus intense que celle qui nous intéresse, mais on pourrait s'en débarrasser par des astuces de polarisation.

Lignes et points singuliers. - J'abandonne maintenant ces nématiques à deux dimensions qui, pour le moment, ont un caractère purement conjectural et je passe à d'autres effets liés à l'existence des variables de phase ou, si vous voulez, d'un groupe continu de symétrie. Si j'étudie les variables de phase $\varphi(r)$ en fonction du point d'observation $r$, elles peuvent avoir des singularités (alors que le paramètre d'ordre luimême, Ia fonction $\mathbf{M}(r)$ ou la fonction $\psi(r)$ ou la fonction $Q_{a \beta}(r)$ n'ont pas de singularités). Et ces singularités peuvent être de différents types. Là encore, nous manquons au stade actuel d'une analyse formelle qui relie la structure des groupes concernés à la nature des singularités.

Ce qu'on connaît, d'une façon, tout à fait empirique, en regardant cas par cas, c'est le catalogue suivant :

a) LIGNES SINGULIÈRES. - On peut caractériser une telle ligne par la propriété suivante. Prenons par exemple un matériau superfluide. Je pars d'un point $P$ et je suis un contour $C$ qui entoure la ligne, je regarde la phase $\varphi(r)$. Eh bien, au lieu de retrouver la même phase, je m'aperçois qu'elle est augmentée de $2 \pi$. J'ai bien retrouvé le même état physique, mais il y a évidemment une singularité si je me rapproche de la ligne (si je rétrécis de plus en plus mon contour). Ceci est connu dans les superfluides (lignes de vortex) et aussi dans les matériaux magnétiques (lignes de Bloch).

Dans le cas des nématiques, nous avons tout à fait l'analogue; nous avons des lignes singulières et, en fait, ce sont ces lignes qui ont donné son nom à la phase. Nématique, vient du grec «nema» qui veut dire «filament 》; ce nom (dû à Georges Friedel) reflète le fait que, quand on regarde une préparation, on voit en général à l'intérieur de la préparation des filaments mobiles. Chacun de ces filaments est une ligne singulière communément appelée « ligne de disclination ». Comme le tenseur diélectrique ou l'ellipsoïde des indices n'est pas le même en chaque point autour de la ligne, le voisinage de celle-ci se comporte un peu comme un prisme et donne des effets de réfraction optique qui rendent la ligne observable.
Vous en verrez tout à l'heure dans le film que j'espère passer à la fin de cet exposé.

Dans les nématiques, l'état représenté par un vecteur $\mathbf{n}$ est identique à l'état représenté par le vecteur - n; pour retrouver le même état physique au bout d'un tour, on n'a pas besoin de tourner de $2 \pi$, il suffit de tourner de $\pi$. Donc, il existe des lignes de disclination pour lesquelles on tourne de $\pi$ seulement : et ceci est associé au fait qu'un paramètre d'ordre est un tenseur de rang 2.

b) Autres singularités. - On pourrait penser à des surfaces de discontinuité. Mais nous n'en observons dans aucun de ces systèmes: les surfaces de discontinuité sont toujours instables par rapport aux lignes. Il n'y a jamais de surfaces discontinues ni dans un ferromagnétique de Heisenberg, ni dans un supraconducteur, ni dans un nématique (dans un ferromagnétique réel, il y a des objets à plus grande échelle que l'on appelle les parois de Bloch, mais une paroi de Bloch n'existe qu'en présence d'anisotropie, c'est-à-dire quand il n'y a plus le groupe continu de symétrie). Donc pas de surface singulière. Par contre, on peut avoir des points singuliers. Dans le cas des ferromagnétiques, il existe peut-être quelquefois des points singuliers. (Cette propriété a été discutée en particulier récemment par Nabarro.) Dans le cas du paramètre d'ordre complexe, des supraconducteurs, je ne suis pas arrivé à imaginer une situation exhibant un point singulier. Dans le cas d'un nématique, on peut en imaginer. La figure 3 vous montre ceci : une tranche de nématique est placée sur un support solide. Au-dessus du nématique, il y a de l'air. C'est donc la surface libre qu'on a ici. Et j'ai choisi le cas où, par exemple, le support solide aime imposer au vecteur $\mathbf{n}$ d'être vertical à son contact. (Très souvent, un support

\section{AIR}

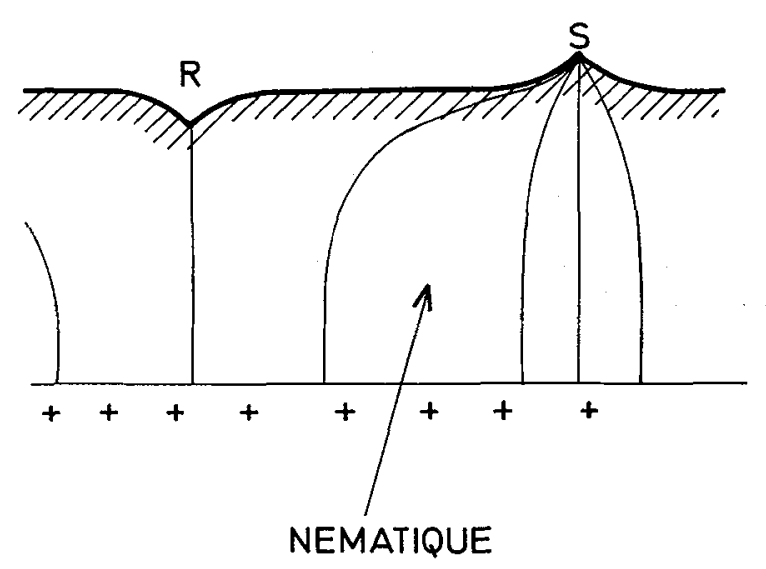

FIG. 3. - Apparition de points singuliers à la surface libre d'un nématique : pour le cas représenté ici, les molécules sont tangentes à l'interface nématique-air. Par contre, par un traitement convenable, on s'est arrangé pour que les molécules soient normales à l'interface verre-nématique. 
solide impose des directions privilégiées et cette propriété est très utile pour faire ce qu'on pourrait appeler des monocristaux nématiques.)

Ici, j'ai choisi une situation volontairement cornélienne dans laquelle le support $\mathrm{du}$ bas voudrait avoir les molécules verticales et, par contre, l'interface air-nématique aime avoir les molécules horizontales.

Que fait le nématique?

Les molécules vont résoudre leur dilemne en faisant apparaître des points singuliers. $\mathrm{Si}$ on analyse ce problème plus en détail, on voit que ces points correspondent à des rugosités de la surface : des «volcans» et des «entonnoirs». (Entendons-nous; il s'agit de volcans à l'échelle qui nous intéresse, c'est-àdire que la hauteur de mon volcan fait un micron.) Idéalement, dans les calculs théoriques, ce système devrait former un réseau régulier de volcans et d'entonnoirs à la surface.

En fait, au stade où nous en sommes, on n'a pas vu de réseau régulier; mais l'existence des points singuliers a été montrée à Harvard par R. B. Meyer. Là encore, l'existence de ces points est finalement reliée de façon très profonde au groupe de symétrie.

Rupture de symétrie par un champ extérieur. - Je vais commencer par parler d'un champ magnétique $H$. Les noyaux benzéniques sont fortement diamagnétiques et ils aiment se placer de façon à déformer les lignes de flux au minimum, c'est-à-dire que le noyau benzénique se met avec le champ dans son plan.

Les molécules nématiques usuelles ont deux noyaux benzéniques qui ne sont pas dans le même plan. L'intersection des deux plans est parallèle à l'axe long de la molécule ; pour que les deux noyaux soient simultanément satisfaits, en présence de $H$, il faut que le champ soit le long de l'axe de la molécule : il $\mathrm{y}$ a tendance à mettre le vecteur $\mathbf{n}$ parallèle au champ. L'énergie diamagnétique impliquée, si on la comptait par molécules, serait quelque chose de ridiculement petit $\left(\sim 10^{-6} k_{B} T\right)$. Mais il faut voir qu'ici, nous n'avons pas une molécule qui tourne, mais une phase ordonnée avec $10^{22}$ molécules qui tournent ensemble. Donc, quoique l'énergie de couplage soit très faible, elle arrive à imposer sa loi : le champ magnétique rompt la symétrie de rotation et impose une direction d'alignement. Il l'impose admirablement s'il est seul à jouer.

Evidemment, on est tout de suite tenté de chercher des situations où un autre effet s'oppose à lui et où le drame peut apparaître. Une telle situation est représentée sur la figure 4. La paroi solide, par un traitement convenable $(*)$, impose aux molécules nématiques d'être parallèles à $\mathrm{OZ}$. Sur la paroi, les molécules doivent être verticales. Nous mettons un champ qui, lui, est parallèle à $\mathrm{OX}$. Il y a alors un ajus-

(*) Il s'agit par exemple d'une paroi de verre polie mécaniquement dans la direction $\mathrm{OZ}$. tement qui se produit par compétition de deux énergies ; l'énergie d'anisotropie diamagnétique et l'énergie de torsion (la même qui intervenait dans les fluctuations dont je parlais tout à l'heure); les deux sont antagonistes et se mettent d'accord pour réaliser une zone de transition avec une certaine épaisseur $\xi(H)$ qui est inversement proportionnelle au champ

$$
\xi(H)=\frac{C}{H} .
$$

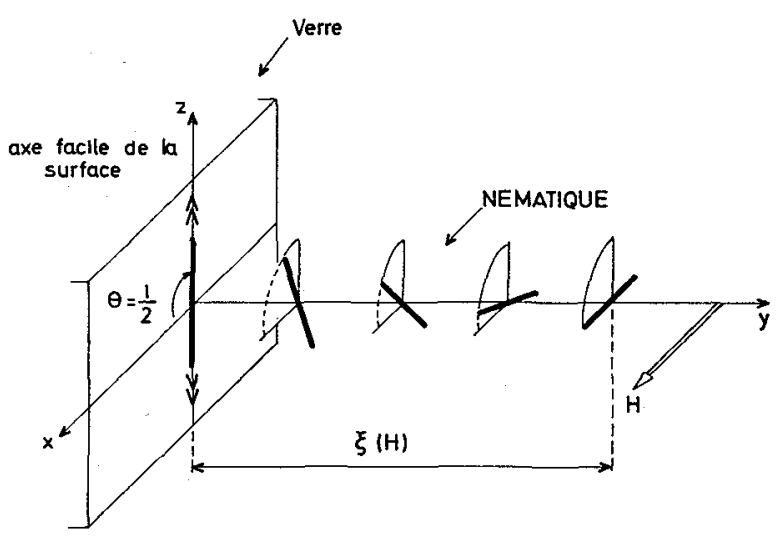

Fig. 4. - Compétition entre alignement par une paroi frottée (selon $\mathrm{Oz}$ ) et par un champ magnétique $\boldsymbol{H}$. La figure représente un cas où $H$ est perpendiculaire à $\mathrm{Oz}$ (torsion globale imposée : $90^{\circ}$ ).

Typiquement, pour $10000 \mathrm{G}$, cette épaisseur vaut quelques microns. Ici, nous touchons du doigt à un ordre de grandeur qui est très typique des cristaux liquides; avec des agents extérieurs assez faibles (ici un champ magnétique, très mal couplé par une faible susceptibilité diamagnétique), nous sommes capables de produire des effets qui peuvent être observés au microscope.

Vous voyez sur cet exemple qu'un matériau nématique est, disons, très flexible optiquement. Il a des propriétés optiques étonnantes et ces propriétés sont très sensibles aux agents extérieurs. Finalement, c'est pour cette raison que ces matériaux sont intéressants.

Sur la figure 4, j'avais imposé une torsion $\theta$ de $90^{\circ}$. Supposez que j'insiste, je tourne la lame de verre dans son plan (angle $\theta$ un peu plus grand que $90^{\circ}$ ). J'aboutis à une situation qui est métastable (le système aimerait mieux avoir une torsion un peu plus petite que $90^{\circ}$ ). Toutefois, cette situation est encore possible et on voit se former progressivement, en faisant tendre $\theta$ vers $180^{\circ}$, une « paroi de Bloch à $180^{\circ}$ » (dont l'épaisseur est encore essentiellement $\xi(H))$. Quand $\theta$ approche de $180^{\circ}$, la paroi est de plus en plus repoussée par l'interface verre/nématique.

Shtrinkman a remarqué que les équations d'équilibre n'ont pas de solution pour $\theta \geqslant 180^{\circ}$. En milieu semi-infini, on ne peut pas enrouler le nématique par plusieurs tours. Ce fait était très regrettable parce que du point de vue optique, on peut imaginer 
des tas de choses intéressantes si on « torsade » fortement le matériau.

$Y$ a-t-il un moyen de violer cette règle en procédant de façon dynamique? Par exemple, partons de $\theta=\pi-\varepsilon$ et imposons brusquement $\theta=\pi$. Comment le système va-t-il relaxer sa torsion ? Il semble qu'il ait plusieurs façons de le faire.

a) Une des façons (la plus fréquente si on opère mal) consiste à faire apparaître une «boucle de disclination »; c'est-à-dire une de ces lignes singulières que je mentionnais tout à l'heure, ici sous forme de boucle fermée, et qui crée une torsion moins forte de $\pi$ à l'intérieur de la boucle qu'à l'extérieur. Cette boucle se met à croître ; finalement elle phagocyte tout le spécimen et relaxe la torsion. Ceci est un mécanisme très familier aux gens qui s'occupent de lignes singulières dans les solides (dislocations) ; c'est un mécanisme mal contrôlé parce que la boucle se forme sur des défauts à la surface du verre.

b) Nous avons fait un acte de foi, nous nous sommes dit que, si on travaille proprement, ou si on a de la chance (dans notre cas il fallait probablement plutôt compter sur la chance) on peut éviter les boucles. Dans ces conditions, il se peut que la paroi se mette à migrer vers le haut, sous l'action de forces qui sont faibles mais parfaitement reproductibles. J'explique maintenant ces deux adjectifs :

(i) La seule force qui agisse sur la paroi est le couplage avec son "image " au-dessous de l'interface verre/nématique. Et comme la partie longue distance des distorsions de paroi est exponentiellement faible $\left(\sim \mathrm{e}^{-z / \xi(H)}\right)$ toutes ces forces images sont petites dès que la distance $Z$ entre paroi et interface est très supérieure à $\xi$.

(ii) Donc la paroi sent une force très faible qui la repousse, mais cette force est très bien définie. Dans un ferromagnétique, nous savons qu'une paroi de Bloch est toujours ancrée sur des défauts du spécimen. Dans un liquide, il n'y a rien de tel ; la symétrie de translation est idéale (à l'échelle macroscopique).

L'expérience a été faite tout récemment par Liliane Léger avec une épaisseur de matériau de l'ordre de $50 \mu$ sous un champ $H \sim 8000 \mathrm{G}$. On tourne le support d'un angle $\theta \approx \pi$ et on voit (quelquefois une minute ou une minute et demie après) apparaître tout à coup, un changement d'éclairement sur la face supérieure qui vous annonce qu'une paroi est arrivée.

Le temps de parcours est très long et dépend énormément du champ parce que, comme je l'ai dit, la force image fait intervenir $\mathrm{e}^{-z / \xi}$ où $\xi$ est cette épaisseur de paroi et $z$ la distance à l'interface. On peut changer $\xi$ en changeant $H$; finalement on a des temps de parcours qui varient exponentiellement avec le champ. Par des changements de champ magnétique assez faibles, disons de 5000 à $8000 \mathrm{G}$, on peut faire varier le temps de migration depuis quelques secondes jusqu'à des minutes, peut-être jusqu'à la dizaine de minutes. Vous devinez immédiatement ce que nous avons envie de faire après : c'est d'imposer une rotation à vitesse uniforme $(\theta=\omega t)$ (ceci étant pour ceux parmi vous qui aiment la supraconductivité un peu l'analogue de l'effet Josephson alternatif : on impose une différence de phase linéaire en temps).

$\mathrm{Si}$ nous parvenons à éviter tous les phénomènes parasites tels que la nucléation de boucles, ce qui doit se passer, c'est qu'on émet un «train de parois" qui monte vers le haut. Les parois ne sont pas équidistantes : chaque paroi est repoussée par ses voisines. Pour la convaincre de monter, il faut qu'elle soit plus près de celle $d u$ bas que de celle du haut. A ce moment-là, elle sent une répulsion plus forte du bas, donc elle monte vers le haut. Nous commençons à voir des trains de paroi sortir mais nous n'avons pas encore vu l'effet le plus spectaculaire qui serait le suivant. Dans des conditions favorables, on devrait pouvoir entasser suffisamment de parois dans le spécimen pour arriver à ce que la distance entre parois soit comparable à une longueur d'onde optique : on aurait alors un réseau presque périodique donc une réflexion de Bragg dans le domaine optique ; c'est-à-dire que ce matériau qui est au départ transparent devrait se colorer par rotation. Pour le moment, cette expérience n'a pas marché mais nous avons encore des espoirs.

Effets d'un champ électrique. - De même qu'il y avait une anisotropie de súsceptibilité magnétique, il y a évidemment une anisotropie de constante diélectrique dans un matériau nématique et ceci devrait imposer certains alignements préférentiels. Par exemple, avec les molécules citées sur la figure 2, la constante diélectrique statique est plus grande si la molécule est perpendiculaire au champ. On s'attend donc à ce qu'elle aime s'aligner perpendiculairement au champ. Eh bien, cette prédiction n'est pas toujours correcte! Les expériences les plus importantes dans ce domaine ont été initiées par G. Heilmeier à RCA. On travaille avec un film nématique (d'épaisseur typique $50 \mu$ ) entre deux électrodes semi-transparentes ; on applique un voltage $V$ entre les électrodes :

a) Pour $V \approx 5 \mathrm{~V}$, la prédiction naïve est bien confirmée : avec le signe choisi plus haut pour l'anisotropie diélectrique, on a un monocristal nẻmatique orienté perpendiculairement au champ électrique. Eclairé en lumière oblique, ce monocristal donne seulement une réflexion spéculaire dans une direction bien définie.

b) Si on monte un peu plus le voltage (disons si on arrive à $8 \mathrm{~V}$ ) tout à coup l'aspect change complètement, le désordre s'établit, on a des écoulements turbulents et, du fait que l'alignement est devenu très désordonné, on a une diffusion de la lumière dans toutes les directions. Ceci, du point de vue d'un observateur qui capte la lumière sortant dans une direction différente du faisceau incident, est très spectaculaire : il avait un fond noir au début et puis il se met à recueillir une 
lumière très intense, c'est-à-dire que le matériau fournit un effet électro-optique très puissant. Il le fournit avec des conditions qui sont extrêmement économiques (sous quelques volts); nous avons des conductivités qui sont dans le domaine des $10^{-8} \Omega^{-1} /$ $\mathrm{cm}^{-1}$; c'est-à-dire que la dissipation en énergie est négligeable, et nous avons des épaisseurs de spécimen qui représentent très peu de matière $(50 \mu$ d'épaisseur). Donc, ici, une situation qui est intéressante du point de vue des applications, qu'il s'agisse d'affichage ou peut-être éventuellement de télévision.

En fait, l'application télévision rencontre encore des difficultés techniques très sérieuses. Mais il y a naturellement un vif intérêt pour ces effets dans les compagnies électroniques ; c'est grâce à cet intérêt que nous trouvons de l'argent pour étudier ces matériaux.

Le mécanisme fondamental impliqué a mis assez longtemps à être élucidé. La première idée, jadis lancée de façon assez vague par un physicien soviétique (V. Zvetkov) et par un physicien américain (E. F. Carr) a été rendue numérique et précise par un physicien allemand, Wolfgang Helfrich. Elle est expliquée sur la figure 5 .
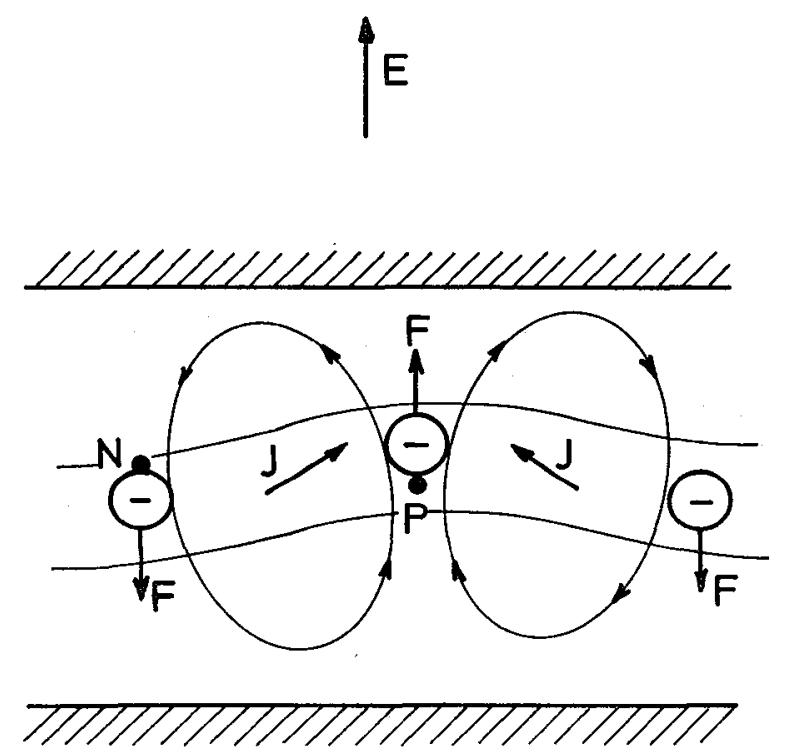

Fig. 5. - Origine de l'instabilité convective dans un spécimen nématique sous champ électrique $E$. Au départ, les molécules sont alignées parallèlement aux électrodes. On a représenté une situation légèrement distordue : les lignes ondulées définissent la direction locale de l'axe optique. C'est ce type de distorsion qui va se trouver amplifiée.

Nous partons de la situation bien alignée et puis nous regardons une petite fluctuation de cet alignement (le même genre de fluctuation que nous décrivions dans la diffusion de la lumière) à une dimension; nos molécules sont un peu tordues dans leur alignement. Ici, intervient l'idée de Carr et Zvetkov; nous savons (de façon purement empirique) que dans ces matériaux la conductance électrique est un peu plus grande dans la direction de l'axe optique que perpendiculairement à cet axe. Alors, si le champ électrique est vertical, il apparaît des composantes horizontales de courant. Ces courants vont faire empiler des charges positives en $\mathbf{P}$ et négatives en $\mathrm{N}$.

Il va en résulter une force volumique (produit de la charge par le champ) qui tend à entraîner le liquide et à établir des mouvements de convection : ces mouvements tendent à faire tourner les molécules et à augmenter la distorsion initiale, d'où une instabilité. La situation est comparable à ce qu'on connaît dans les instabilités thermiques d'une couche chauffée par en bas. On obtient ainsi des rouleaux de convection puis, sous des champs plus élevés, un régime turbulent. Ces différents effets sont très bien illustrés dans le film que nous allons projeter maintenant. Ce film a été réalisé par $\mathrm{R}$. Kashnow (General Electric) qui a travaillé en collaboration assez étroite avec nous sur ces questions.

A travers ces propos très fragmentaires, j'espère que vous voyez un peu se dessiner la perspective d'ensemble des recherches sur les cristaux liquides: voici une branche assez ancienne de la physique, illustrée jadis en France par Mauguin, Grandjean, G. Friedel, mais étudiée seulement par de très petites équipes depuis la guerre. Tout à coup elle reprend une vitalité remarquable et de nombreux groupes s'y consacrent. Mais il s'agit d'un domaine pluridisciplinaire et touffu: quand on veut $y$ former des étudiants, il faut qu'ils connaissent de la physicochim e et de la chimie, aussi certains aspects de la physique du solide (en particulier les dislocations). Il est bon - il est impératif même - qu'ils connaissent du magnétisme parce qu'il y a beaucoup de rapprochements féconds à faire avec cette science. Finalement, il est nécessaire qu'ils connaissent de l'hydrodynamique assez avancée - phénomènes d'instabilité et de turbulence. Actuellement, nos nouveaux groupes français sont très débutants dans la plupart de ces domaines, mais je pense qu'ils parviendront quand même à trouver des choses amusantes et éventuellement utiles.

Quelques références de base sur les cristaux liquides :

Friedel (G.), Annales de Physique, 1922, 18, 273.

Chistiakov (I. G.), Soviet Physics Uspekhi, 1967, 9, 551. SAupe (A.), Angewandte Chemie (édition anglaise), 1968, 7, 97. 